\title{
Analysis of Fingerprint Recognition Characteristics Based on New CGH Direct Comparison Method and Nonlinear Joint Transform Correlator
}

\author{
Man Ho Jeong* \\ Department of Laser $\&$ Optical Information Engineering, Cheongju University, \\ 36 Naeduk-dong, Sangdang-gu, Cheongju 360-764, Korea
}

(Received September 23, 2009 : revised December 3, 2009 : accepted December 3, 2009)

\begin{abstract}
Fingerprint recognition using a joint transform correlator (JTC) is the most well-known technology among optical fingerprint recognition methods. The JTC method optically compares the reference fingerprint image with the sample fingerprint image then examines match or non-match by acquiring a correlation peak. In contrast to the JTC method, this paper presents a new method to examine fingerprint recognition by producing a computer generated hologram (CGH) of those two fingerprint images and directly comparing them. As a result, we present some parameters to show that fingerprint recognition capability of the CGH direct comparison method is superior to that of the JTC method.
\end{abstract}

Keywords : Joint transform correlator, Computer generated hologram, Finger-print recognition OCIS codes : (090.0090) Holography; (090.1760) Computer holography

\section{INTRODUCTION}

Fingerprint recognition compares input information of a user on a terminal to the user information registered and stored in a local computer or a host computer to judge whether they match.. Recently many methods for accurate fingerprint recognition and perfect security have been studied [1-2]. Among them the joint transform correlator (JTC) method may be the most typical one as an encryption technology using an optical method [3-4]. In addition to that, the essential topic in fingerprint recognition is the technology to find the center point of a fingerprint image and accurately recognize it when it is incorrect in angle and location [5].

An optical fingerprint recognition method JTC uses a type of optical pattern recognition system and it quantitatively analyzes the match or non-match of the fingerprint image by measuring the optical correlation peak and peak signal to noise ratio (PSNR) of the sample fingerprint image, which needs to be compared with the reference fingerprint image. This kind of optical pattern recognition system goes through a double random encryption and double-encryption process using the JTC method. It has the merits that the optical system is relatively simple and can compare samples in real time. However, it also has a shortcoming in that it is

*Corresponding author: manho@cju.ac.kr sensitively affected by turning or moving of fingerprint images. A study of fingerprint recognition improvement using a double random encryption method or an algorithm to find the center point of a fingerprint to solve the recognition problem due to turning and moving of fingerprint images has been already published in another paper [4].

In this paper, we present a new method to examine the fingerprint recognition by producing computer generated holograms $(\mathrm{CGH})$ of those two fingerprint images and directly comparing them, unlike the JTC method which acquires the correlation between the two fingerprint images reconstructed from a Fourier hologram in the form of interference fringes. The reason that this paper presents a new $\mathrm{CGH}$ direct comparison method is that there has been no study conducted using the CGHs of fingerprint images, rather than using the fingerprint images themselves, for comparing and analyzing the characteristics of fingerprints. The widely-used JTC method uses an optical system to compare the images themselves. Therefore, the objective of this paper is to emphasize the merits of the direct CGH comparison method over the JTC method based on the fingerprint experimental results of each. In this paper, we used the non-linear joint transform correlator (NJTC) to solve the problem of the non-linearity of the digital camera in JTC system.

CGH is generally divided into cell-oriented and point-oriented methods. For cell-oriented CGH, the 
Lohmann method and the Lee method are well-known [7-8]. These methods simplify the phase and amplitude information by encoding them to the basic structure of a cell. On the other hand, the point-oriented CHG makes it possible to recreate an image approaching the original object by recording the discrete Fourier transform (DFT) values corresponding to each point of an object. Then the amplitude and phase values of the CGH can be obtained by adding a further mathematical method or repetitive adding processes on the results of the DFT [6,9]. This paper applies point-oriented CGH to fingerprint recognition and especially applies the cosine CGH using a sinusoidal wave, which is the typical method for holographic diffraction gratings.

\section{DIRECT CGH COMPARISON OF FINGERPRINT IMAGES}

This paper presented a new fingerprint recognition system which forms CGHs acquired from the reference image and from the sample image and directly compares those CGHs. The reason for which we presented this method is that other fingerprint recognition systems including NJTC focus on comparing the fingerprint images themselves. Therefore, the objective of this study is to analyze the merits of our new direct CGH comparison method and to compare its fingerprint recognition results with those of the NJTC method. Figure 1 shows the optical structure of the basic NJTC fingerprint recognition system.

Let us call the reference fingerprint image $\mathrm{r}(x, y)$ and the sample fingerprint image $\mathrm{s}(x, y)$ and assume that those two inputs are separated by $2 x_{0}$. Now these two images are Fourier transformed by the Fourier lens L1 and interfered together on the Fourier plane. The information acquired on the Fourier plane is shown as mutually interfered information between the Fourier transformed value $\mathrm{R}(u, v)$ of the reference fingerprint image $\mathrm{r}(x, y)$ and the Fourier transformed value $\mathrm{S}(u, v)$ of the sample fingerprint image $\mathrm{s}(x, y)$. The intensity of this interference fringe pattern is called the joint power spectrum (JPS). Therefore, the intensity of the inter-

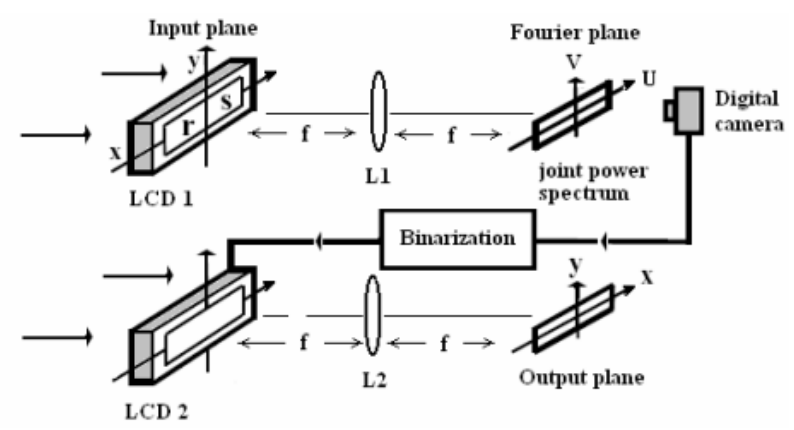

FIG. 1. NJTC fingerprint recognition system. fered light, JPS, acquired with a light detector on the Fourier plane can be expressed as in equation (1)

$$
\begin{aligned}
& |G(u, v)|^{2}=|R(u, v)|^{2}+|S(u, v)|^{2} \\
& +R(u, v) S^{*}(u, v) \exp \left(-j u x_{0}\right)+R^{*}(u, v) S(u, v) \exp \left(+j u x_{0}\right)
\end{aligned}
$$

Here $u, v$ are independent spatial frequency variables scaled by $2 \pi / \lambda f, \lambda$, is the wavelength of input light and $f$ is the focal length of the Fourier transform lens L1. Now, the signal acquired on the inverse Fourier plane, which is the output plane by the inverse Fourier transform lens L2, is expressed as a correlated signal between two signals of the reference fingerprint image and the sample fingerprint image located on the input plane. Equation (2) shows the correlated output signal acquired on the output plane.

$$
\begin{aligned}
& g(x, y)=\left[r(x, y) \odot r^{*}(x, y)\right]+\left[s(x, y) \odot s^{*}(x, y)\right] \\
& +(\lambda f)^{2}\left[s(x, y) \odot r^{*}\left(x+2 x_{0}, y\right)\right]+(\lambda f)^{2}\left[s^{*}(x, y) \odot r\left(x-2 x_{0}, y\right)\right]
\end{aligned}
$$

Here, $\odot$ is the correlation operator. In equation (2), the first and the second terms are the DC terms created in the center of the output signal, and they can be eliminated using a filter. The third and the fourth signals are correlated signals formed at $+2 x_{0}$ and $-2 x_{0}$ apart, respectively, and they play important roles deciding match or non-match between the reference image and the sample image. Finally they are used in calculating the correlation peak.

Now the basic optical structure of the direct CGH comparison system is shown in figure 2. The basic principle of the fingerprint recognition system shown in the figure 2 is to get Fourier transformed images from the Fourier transform lens on the Fourier plane after placing the reference fingerprint image and the sample fingerprint image in set time intervals on the SLM located on the input plane. In other words, the direct CGH comparison method does not place two pieces of image simultaneously but places them with a slight time interval on spatial light modulator(SLM), namely liquid crystal display(LCD) in figure 2. Then these two images are Fourier transformed by the lens and are sent to the computer. Immediately, the Fourier transformed images are calculated in the form of various kinds of CGH's such as cosine CGH and are ready to

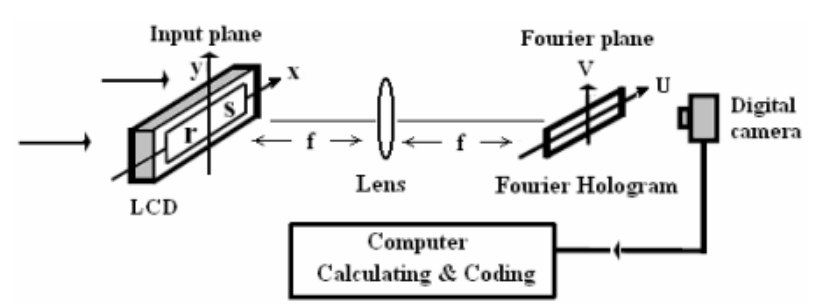

FIG. 2. Direct CGH comparison fingerprint recognition system. 
be compared to judge whether they match.

When we allow the Fourier transformed signal on the Fourier plane to interfere with the cosine reference wave, we can get cosine amplitude and phase CGH. The complex amplitude and phase of the cosine $\mathrm{CGH}$ formed on the Fourier plane can be expressed as Equation (3). However, the cosine CGH here is formed on a two dimensional plane, so the diffraction grating pattern constructed here is assumed to have only a $u$ component, that is to say, the grating line is created parallel to the $\mathrm{v}$ axis [3].

$$
T(u, v)=\frac{1}{2}\left[1+A(u, v) \cos \left(2 \pi f_{0} u+\Phi(u, v)\right)\right]
$$

Here, $\mathrm{A}(u, v)$ is the amplitude of the CGH and $\phi(u, v)$ shows the phase of the CGH. Moreover, $f_{\mathrm{o}}$ is the spatial frequency of the cosine $\mathrm{CGH}$ and $\lambda$ and $f$ are the wavelength of the input light and the focal length of the Fourier transform lens, respectively.

When we analyze the components forming the CGH in the equation (3), it can be expressed like the equation (4).

$$
\begin{aligned}
& T(u, v)=\frac{1}{2}+\frac{A(u, v) \exp (j \Phi(u, v))}{4} \exp \left(j 2 \pi f_{o} u\right) \\
& +\frac{A(u, v) \exp (-j \Phi(u, v))}{4} \exp \left(-j 2 \pi f_{o} u\right)
\end{aligned}
$$

Here, the cosine CGH function $U(u, v)$ can be expressed as equation (5).

$$
U(u, v)=A(u, v) \exp (j \Phi(u, v))
$$

As we explained earlier, the cosine CGH expressed as equation (5) can be obtained by causing the Fourier transformed signal to interfere with the cosine reference signal, as shown in figure 2 , but it can also be obtained through equation (5) after sending the Fourier transformed signals directly to the computer. Now, the Fourier transformed images are calculated in the form of cosine CGH. The reconstructed image $u_{R}(x, y)$ obtained from the cosine CGH is expressed in equation (6).

$$
u_{R}(x, y)=\frac{\delta(x, y)}{2}+\frac{1}{4} u\left(x+x_{o}, y\right)+\frac{1}{4} u^{*}\left(-x+x_{o}, y\right)
$$

In equation (6), the second term represents the real image of the object and the third term shows the virtual image, which is the conjugate wave of the object. Here, $x_{0}$ is the location of the reconstructed image regenerated on the off-axis.

\section{EXPERIMENTAL RESULTS}

To compare the results using the NJTC system shown in figure 1 with the results using the method of direct
CGH comparison, we prepared some fingerprint samples. Various types of $256 \times 256$ binary fingerprint images are shown in figures 3 and 4 . Those fingerprint images were divided into 3 groups: in group A, we used A-1 as the reference fingerprint image, and we partially damaged fingerprints and marked them as A-2, A-3 and A-4 as shown in figure 3 . In group B, we transformed the reference fingerprint image A-1 and changed it to different fingerprints B-1 and B-2 as shown in

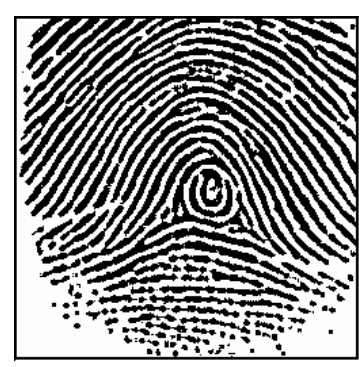

(a)

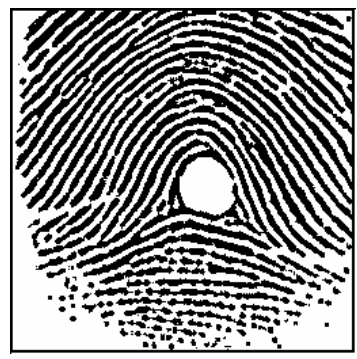

(c)

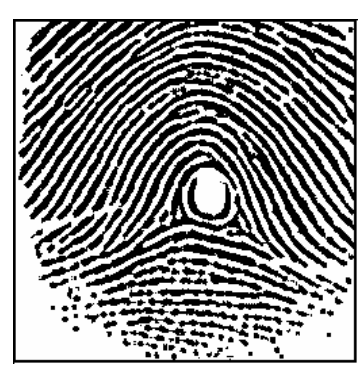

(b)

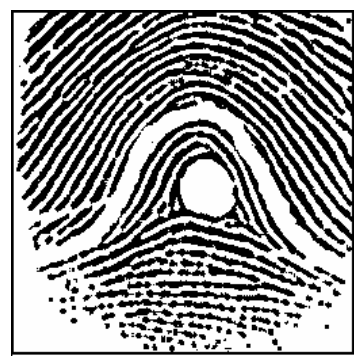

(d)
FIG. 3. 256x256 binary fingerprint images (group A); (a) A-1 (b) A-2 (c) A-3 (d) A-4.

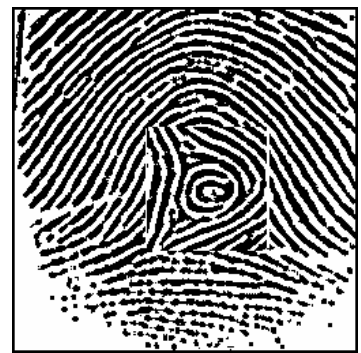

(a)

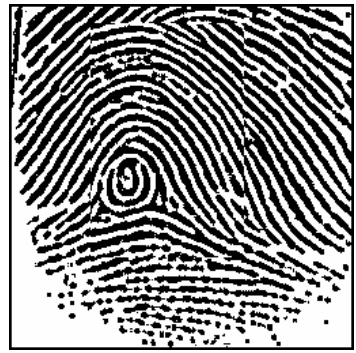

(b)

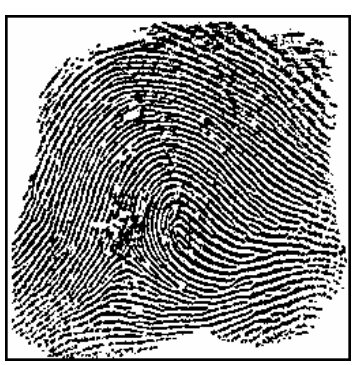

(c)

FIG. 4. 256x256 binary fingerprint images (group B, C); (a) B-1 (b) B-2 (c) C-1. 
figure 4. In group C, we prepared another fingerprint totally different from the reference fingerprint image $\mathrm{A}-1$, and we marked it $\mathrm{C}-1$ and showed it in figure 4 again. For group A, the aim is to find out whether the recognition results are similar. For groups $\mathrm{B}$ and $\mathrm{C}$, it is to expect the fingerprint recognition results much different from group A. However, the reason why we separated groups B from C is to check whether the degree of non-match with the totally different fingerprint image $\mathrm{C}-1$ in group $\mathrm{C}$ is bigger than that with the partially changed images B-1 and B-2 in group B.

First, to evaluate the fingerprint recognition capability using the NJTC fingerprint recognition system, we placed the reference fingerprint image $\mathrm{A}-1$ on the left and the sample fingerprint images A-2 to $\mathrm{C}-1$ on the right and then calculated the correlated peak, averaged noise and the PSNR. The NJTC fingerprint recognition system finally examines the degree of correlation, match or non-match, between the reference fingerprint image and the sample fingerprint image on the output plane by calculating the correlation peak. An important point is that the result obtained on the output plane as shown on equation (2) is a form for two images located on the input plane reconstructed together. Therefore, although it used an optical correlator system, it eventually is a method of comparing the reference fingerprint image and the sample fingerprint image with the correlated maximum value.

Next, we would like to discuss the method of evaluating the degree of fingerprint recognition by directly comparing CGH's as presented in this paper. The importance of this method is that while the NJTC method used the concept of the correlated maximum value and eventually compared the reference fingerprint image with the sample fingerprint image, our method compares CGH's respectively obtained from the reference fingerprint image and the sample fingerprint image using MSE and SNR concepts.

Now, the cosine CGH's obtained from the reference fingerprint image and sample fingerprint image respectively

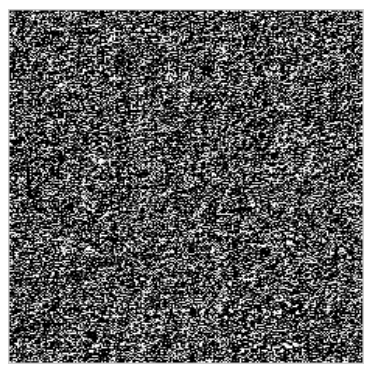

(a)

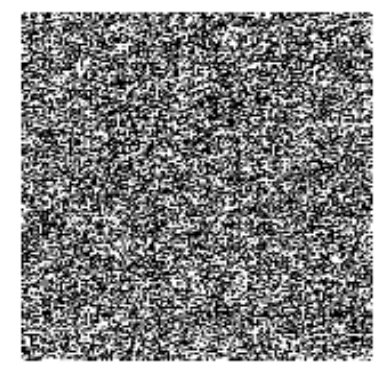

(b)
FIG. 5. CGH obtained from the fingerprint image A-1; (a) amplitude and phase cosine CGH (b) binary phase cosine CGH. have amplitude $A(u, v)$ and phase $\phi(u, v)$. This CGH is called the amplitude and phase cosine CGH. Based on this amplitude and phase cosine $\mathrm{CGH}$, we can encode the phase $\phi(u, v)$ with 0 and 1 by the phase value, and the obtained CGH in this method is called the binary phase cosine CGH. We evaluated the capability of fingerprint recognition by creating the amplitude and phase cosine $\mathrm{CGH}$ and binary phase cosine $\mathrm{CGH}$. Figure 5 shows the amplitude and phase cosine CGH and binary phase cosine $\mathrm{CGH}$ obtained from the reference fingerprint image A-1. Moreover, figures 6(a) and $6(\mathrm{~b})$ respectively show the amplitude and phase cosine CGH and the binary phase cosine CGH obtained from the sample fingerprint image $\mathrm{C}-1$ which is totally different from the reference fingerprint image.

As a scale to quantitatively measure the fingerprint recognition capability of the direct $\mathrm{CGH}$ comparison method, we used MSE and SNR [10] defined as the following.

$$
\begin{aligned}
& M S E=\frac{1}{M N} \sum_{u=0}^{N-1 M-1} \sum_{v=0}^{[-1}\left[U_{r}(u, v)-\left.U_{s}(u, v)\right|^{2}\right] \\
& S N R=\frac{\sum u, v\left[U_{r}(u, v)\right]^{2}}{\sum u, v\left[U_{r}(u, v)-U_{s}(u, v)\right]^{2}}
\end{aligned}
$$

In here, $N \times M$ is the number of pixels in the image, $U_{r}(u, v)$ is the CGH obtained from the reference fingerprint image, and $U_{s}(u, v)$ is the CGH obtained from the sample fingerprint image.

Table 1 shows the correlation peak, PSNR, MSE and SNR of various fingerprint images obtained using the NJTC fingerprint recognition system. In general correlation peak and PSNR are enough for evaluating the performance of the NJTC system, but we added MSE and SNR parameters to compare the ability of the NJTC with our new CGH direct comparison method. The values of MSE and SNR were obtained by using equations (7) and (8), respectively, by comparing reconstructed images of $r(x, y)$ and $s(x, y)$ from the output

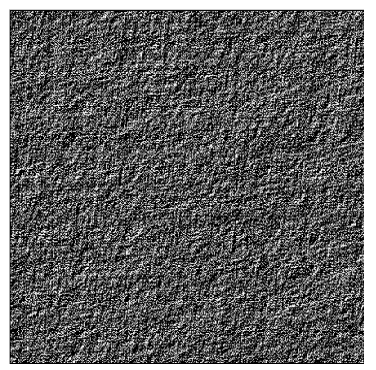

(a)

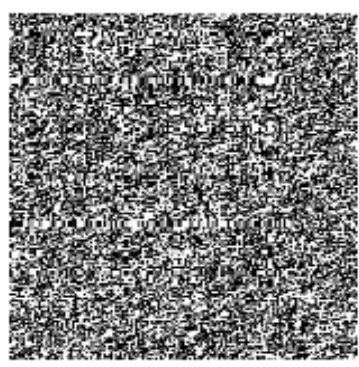

(b)
FIG. 6. CGH obtained from the fingerprint image C-1; (a) amplitude and phase cosine CGH (b) binary phase cosine CGH. 
TABLE 1.Correlation peak, PSNR, MSE and SNR obtained by using the NJTC system

\begin{tabular}{c|c|c|c|c}
\hline \hline sample & corr. peak & PSNR & MSE & SNR \\
\hline A-1 & 14512 & 868749 & 2685.5691 & 1.0292 \\
\hline A-2 & 14486 & 692241 & 2685.6951 & 1.0292 \\
\hline A-3 & 14455 & 629369 & 2685.7001 & 1.0292 \\
\hline A-4 & 14238 & 492069 & 2685.7255 & 1.0292 \\
\hline B-1 & 12442 & 372841 & 2693.0644 & 1.0264 \\
\hline B-2 & 10714 & 255983 & 2698.9946 & 1.0241 \\
\hline C-1 & 6198 & 86106 & 2714.4885 & 1.0183 \\
\hline
\end{tabular}

correlation plane, that is, the inverse Fourier plane as shown in equation (2). Figure 7 shows the three dimensional picture of the correlation peak signal obtained from two representative samples. Figure $7(\mathrm{a})$ is for the sample A-1 which is the same as the reference image, and figure $7(\mathrm{~b})$ is for the sample C-1 totally different from the reference image. Table 2 shows the MSE and SNR values obtained by directly comparing CGH's acquired from the fingerprint images as shown in figure 2 . The values of MSE and SNR shown on table 2 were obtained using equations (7) and (8), respectively, by comparing CGH's instead of reconstructed images. The left values were obtained through the amplitude and phase cosine $\mathrm{CGH}$ and the right ones were obtained through the binary phase cosine CGH.

Let's look at table 1 showing the results of using the NJTC system. When we use A-1 as the sample fingerprint image the same as the reference one, the obtained correlation peak and PSNR is $1.45 \mathrm{e}+004$ and $8.69 \mathrm{e}+005$, respectively. When we use the $\mathrm{C}-1$ as the sample fingerprint image totally different from the reference one, the obtained correlation peak and PSNR is $6.20 \mathrm{e}+003$ and $8.61 \mathrm{e}+004$, respectively. Now, let's look at the right side of table 1 to estimate the MSE and SNR of the NJTC system. The obtained MSE and SNR for the sample A-1 is 2685.5691 and 1.0298, respectively. Next, the obtained MSE and SNR for the sample C-1 is 2714.4885 and 1.0183 , respectively. The results concerning about the correlation peak and PSNR for the NJTC system indicate that the ratio of correlation peak is a maximum of 2.34 and the ratio of PSNR is a maximum of 10.08. Again the results concerning about the MSE and SNR for the NJTC system indicate that the ratio of MSE is a maximum of 1.01 and the ratio of SNR is a maximum of 1.01 .

Now, let us look at table 2 showing the results of using the direct CGH comparison method. First, let's look at the left side of table 2 representing the case using the amplitude and phase CGH. For the sample A-1, the obtained MSE and SNR is 2.50 and 205.05, respectively. On the other hand, for the sample C-1 the obtained MSE and SNR is 404.69 and 1.27, respec-

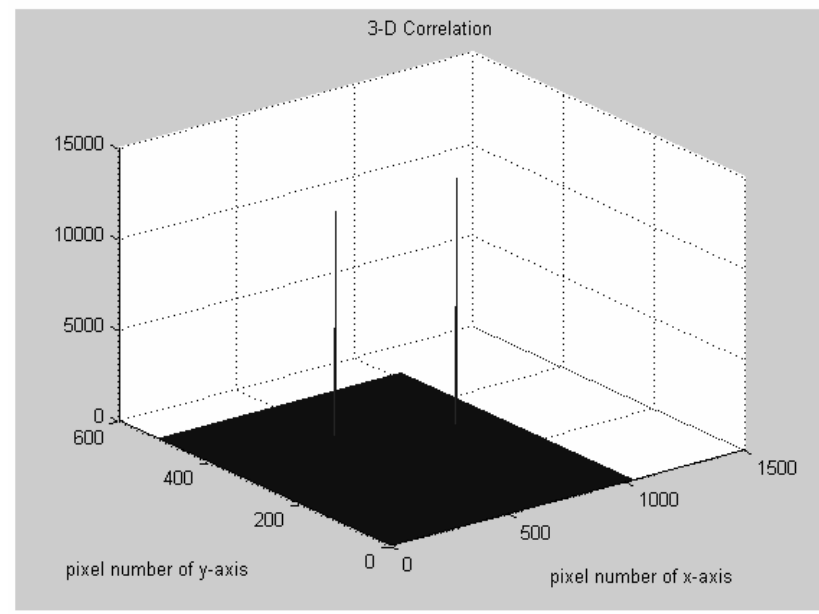

(a)

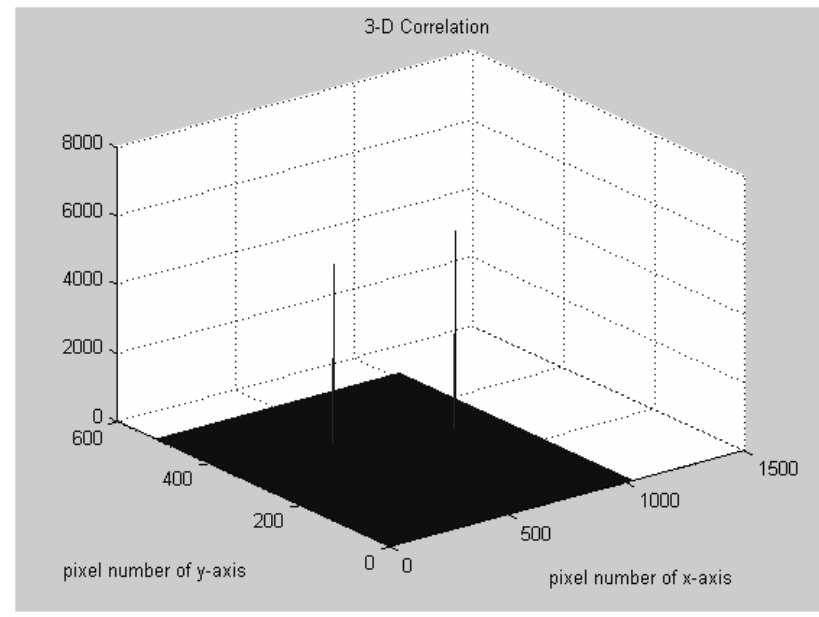

(b)

FIG. 7. Three dimensional correlation peak signal; (a) match case using A-1 as the input fingerprint image (b) non-match case using $\mathrm{C}-1$ as the input fingerprint image.

TABLE 2. MSE and SNR obtained by using the direct CGH comparison system

\begin{tabular}{c|r|r|r|r}
\hline \hline \multirow{2}{*}{ sample } & \multicolumn{2}{|c|}{$\begin{array}{c}\text { ampitude and phase } \\
\text { cosine CGH }\end{array}$} & \multicolumn{2}{c}{ binary phase CGH } \\
\cline { 2 - 5 } & \multicolumn{1}{c}{ MSE } & \multicolumn{1}{c}{ SNR } & MSE & \multicolumn{1}{c}{ SNR } \\
\hline A-1 & 2.5010 & 205.0497 & 10.1020 & 396.2542 \\
\hline A-2 & 4.2500 & 120.8363 & 20.8570 & 172.9845 \\
\hline A-3 & 8.7637 & 58.5881 & 30.2303 & 118.8891 \\
\hline A-4 & 31.8889 & 16.1015 & 56.4012 & 63.7607 \\
\hline B-1 & 78.3878 & 6.5516 & 91.4591 & 39.5161 \\
\hline B-2 & 153.5508 & 3.3439 & 129.0623 & 28.1130 \\
\hline C-1 & 404.6944 & 1.2688 & 207.6729 & 17.3914 \\
\hline
\end{tabular}

tively. Thus for the direct CGH comparison method concerning about using the amplitude and phase CGH, the results indicate that the ratio of MSE is a maximum of 161 and the ratio of SNR is also a maximum of 161 . Next, let's look at the right side of table 2 representing 
the case using the binary phase CGH. For the sample A-1 the obtained MSE and SNR is 10.10 and 396.25, respectively. On the other hand, for the sample C-1 the obtained MSE and SNR is 207.67 and 17.39, respectively. Thus for the direct CGH comparison method concerning about using the binary phase $\mathrm{CGH}$, the results indicate that the ratio of MSE is a maximum of 20.56 and the ratio of SNR is a maximum of 22.79 .

When we compare the fingerprint recognition results using the NJTC method and the direct CGH comparison method, the direct CGH comparison method is about 68 times superior to the NJTC method when we use the amplitude and phase CGH, and about 8.8 times superior when we use the binary phase CGH. One interesting fact is that the method using the amplitude and phase $\mathrm{CGH}$ is about 7.7 times superior to the method using the binary phase CGH. By checking the MSE and SNR value of the NJTC as shown on the right side of table 1 , we can find another interesting fact that it is difficult to distinguish the match between the samples because the results of MSE and SNR indicate only a small difference in values. Furthermore, another important fact can be find out through the result shown in table 1 and table 2 . When we look at the result of using fingerprints B-1 and B-2, which are classified as different fingerprints than the reference fingerprint A-1 by greatly changing the fingerprint, the direct CGH comparison method shows much superior capability in match or non-match distinction to the NJTC method. Finally, when we look at the result of using A-2, A-3 and A-4 as sample fingerprint images, which were obtained by decreasing the information of the reference fingerprint image A-1 a little, we can conclude that for the case of using direct CGH comparison it recognizes them as one person's fingerprint; however, for the sample A-4 which has greater damage, it is difficult to be sure that it recognizes the two as the same fingerprint. On the other hand, for the case of using the NJTC, the differences of the correlation peak between the A type samples is not enough to distinctly determine a match or non-match. Moreover we can mention the same conclusion when MSE and SNR parameters are used instead of correlation peak and PSNR.

\section{CONCLUSION}

In this paper, we presented a new direct CGH comparison method of fingerprint recognition. This method examines match or non-match by directly comparing
CGHs of fingerprint images, unlike the NJTC method which ultimately uses the fingerprint images reconstructed from the Fourier hologram on the Fourier plane. We analyzed its fingerprint recognition capability by comparing it with the fingerprint recognition method using NJTC. By looking at the result of the NJTC and the direct CGH comparison method, we found out the direct CGH comparison method is about 68 times superior to the NJTC method when using the amplitude and phase CGH, and about 8.8 times superior when using the binary phase CGH. Furthermore, the method using the amplitude and phase CGH is 7.7 times superior to the method using the binary CGH. Therefore, the direct CGH comparison method presented in this paper is an effective method with superior fingerprint recognition capability. Further studies should be conducted on improving the accuracy of the fingerprint recognition by analyzing the damaged fingerprint images accurately.

\section{REFERENCES}

1. H. C. Lee and R. E. Gaensslen, Advances in Finger-print Technology (Elsevier, New York, USA, 1991).

2. A. Jain, L. Hong, and R. Bolle, "On-line fingerprint verification," IEEE Trans. Pattern Analysis and Machine Intell. 19, 302-314 (1997).

3. J. W. Goodman, Introduction to Fourier Optics, 2nd ed. (McGraw Hill, New York, USA, 1996), pp. 79-87.

4. S. B. Kim, S. H. Joo, and M. H. Jeong, "A study on correlation-based fingerprint recognition method, Hankook Kwanghak Hoeji (Korean J. Opt. Photon.) 13, 493-500 (2002).

5. A. M. Bazen and S. H. Gerez, "Directional field computation for fingerprints based on the principal component analysis of local gradients," in Proc. RISC 2000, 11th Annual Workshop on Circuits, Systems and Signal Processing (Veldhoven, The Netherlands, 2000).

6. H.-J. Yang, J.-S. Cho, and Y.-H. Won, "Reduction of reconstruction errors in Kinoform CGHs by modified simulared annealing algorithm,” J. Opt. Soc. Korea 13, 92-97 (2009).

7. A. W. Lohmann and D. P. Paris, "Binary Fraunhofer holograms generated by computer," Appl. Opt. 6, 1739-1748 (1967).

8. W. H. Lee, "Sampled Fourier transform holograms generated by computer," Appl. Opt. 9, 639-643 (1970).

9. F. Wyroski and O. Bryngdahl, "Iterative Fourier transform algorithm applied to computer holography," J. Opt. Soc. Am. 5, 1058-1065 (1988).

10. B. V. K. V. Kumar and L. Hassebrook, "Performance measure for correlation filters," Appl. Opt. 29, 2997-3006 (1990). 